\title{
Lewis-Acid Doping of Triphenylamine-Based Hole Transport Materials Improves the Performance and Stability of Perovskite Solar Cells
}

Jiang Liu, * Wenzhu Liu, Erkan Aydin, George T. Harrison, Furkan Halis Isikgor, Xinbo Yang, Anand Selvin Subbiah, Stefaan De Wolf,*

KAUST Solar Center (KSC), Physical Sciences and Engineering Division (PSE), King Abdullah University of Science and Technology (KAUST), Thuwal 23955-6900, Kingdom of Saudi Arabia.

* Corresponding author

E-mail address: jiang.liu@,kaust.edu.sa; stefaan.dewolf@kaust.edu

\begin{abstract}
:
Highly efficient perovskite solar cells (PSCs) fabricated in the classic $n-i-p$ configuration generally employ triphenylamine-based hole-transport layers (HTL) such as spiro-OMeTAD, PTAA, poly-TPD. Controllable doping of such layers has been critical to achieve increased conductivity and high device performance. To this end, LiTFSI/tBP doping and subsequent air exposure is widely utilized. However, this approach often leads to low device stability and reproducibility. Departing from this point, we introduce the Lewis acid tris(pentafluorophenyl)borane (TPFB) as an effective dopant, resulting in a significantly improved conductivity and lowered surface potential for triphenylamine-based HTLs. Here, we specifically investigated spiro-OMeTAD, which is the most widely used HTL for $n-i-p$ devices, and revealed improved PCE and stability of the PSCs. Further, we demonstrated the applicability of TPFB doping to other triphenylamine-based hole transport layers (HTLs). Spectroscopic characterizations reveals that TPFB doping results in significantly improved charge transport and reduced recombination losses. Importantly, the TPFB-doped perovskite devices retained near $85 \%$ of initial PCE after 1000 hours of storage in the air, while the conventional LiTFSI-doped device dropped to $75 \%$. Finally, we give insight to utilize other similar molecular dopants such as fluorine-free triphenylborane (TPB) and phosphorus-
\end{abstract}


centered tris(pentafluorophenyl)phosphine (TPFP) by density functional theory (DFT) analysis underscoring the significance of the central boron atom and fluorination in TPFB for the formation of Lewis acid-base adducts.

Keywords: Perovskite; Lewis acid; Triphenylamine; Doping; Solar cell; Stability

\section{Introduction}

Metal halide perovskite-based photovoltaic devices have seen an unprecedented rise in performance over the past decade, with their certified power conversion efficiencies (PCEs) recently reaching over $25 \%{ }^{1}$. These perovskite materials possess excellent optical absorption properties, even close to their band edge, thereby requiring only a few hundred nanometers of film to fully absorb the desired spectrum ${ }^{2-3}$. Both theoretical and experimental results have confirmed that the perovskite materials exhibit energetically shallow intrinsic defects and electronically benign grain boundaries ${ }^{4-5}$. Besides the quality of the perovskite material, the choice of charge transport layers also plays a significant role in the photovoltaic device performance ${ }^{6-7}$. In the field of perovskite solar cells (PSCs), for either $n-i-p$ or $p-i-n$ device configurations, $p$-type organic hole-transport layers (HTLs) have been commonly used due to their convenience of deposition. However, organic semiconductors generally suffer from low intrinsic carrier concentration and therefore low conductivity. Additionally, in the classical $n$ $i-p$ configuration, a certain minimal thickness of solution-processed organic HTLs is required to fully cover the underlying perovskite layer, which mandates some methods to improve their conductivity for efficient charge transport. In this context, extrinsic $p$-type doping of such organic layers may be a successful strategy.

Indeed, the use of doped organic transport layers in PSCs may facilitate charge transport, reduce the sensitivity of their performance to the electrode work function, and also improve the device efficiency ${ }^{8-9}$. Dopants for HTLs reportedly include ionic liquid ${ }^{10}, \mathrm{FeCl}_{3}{ }^{11}, \mathrm{~V}_{2} \mathrm{O}_{5}{ }^{12}$, 2,3,5,6-tetrafluoro-7,7,8,8-tetracyanoquinodimethane (F4TCNQ) ${ }^{9}$, cobalt-complexes ${ }^{13}$ and the more widely used lithium bis(trifluoromethanesulfonyl)imide (LiTFSI) ${ }^{14-17}$; most of these dopants are not easy to process and may lead to device-stability issues. For instance, although 
F4TCNQ is a frequently used dopant, its solubility in common solvents such as chlorobenzene (CB) is very low ${ }^{18}$. LiTFSI has similar solubility issues and is usually pre-dissolved in acetonitrile. In addition, liquid 4-tert-butylpyridine (tBP) is also required to further improve the solubility of LiTFSI and promote the homogeneous distribution of Li salt in spiro$\mathrm{OMeTAD}^{19-21}$. However, tBP is detrimental to the underlying perovskite layer and causes adverse morphological changes after evaporation ${ }^{21-23}$. Although LiTFSI cannot directly oxidize spiro-OMeTAD, it facilitates the process in the presence of oxygen; the large $\mathrm{TFSI}^{-}$anion stabilizes the oxidation states via formation of spiro-OMeTAD ${ }^{+}$TFSI $^{-} .{ }^{14}$ Upon successful completion of the doping process, spiro-OMeTAD oxidizes to spiro-OMeTAD ${ }^{+}$, which, however, is highly sensitive to light, humidity, and oxygen ${ }^{14-15}$, causing reproducibility problems. Moreover, since LiTFSI is hygroscopic and tends to absorb moisture present in the air, LiTFSI doping may accelerate the degradation of metal halide perovskites ${ }^{24-25}$. Therefore, exploring novel doping strategies for organic HTLs is critical to overcome the challenges described above.

Earlier, TPFB has been shown to be an effective dopant for some conjugated polymers ${ }^{8,26-}$ ${ }^{27}$, resulting in increased charge mobility and density. Ye, et al. ${ }^{28-29}$ have demonstrated the use of TPFB in spiro-OMeTAD and PTAA materials, but the device performance is still relatively low. In the case of conventional LiTFSI doping, due to their small atomic radius and hence high diffusivity, $\mathrm{Li}^{+}$ions can diffuse across the perovskite layer to accumulate in the ETL, adversely affecting device performance and resulting in hysteresis. ${ }^{30}$ Some methods have been proposed to solve these problems in HTL doping. For example, Liu et al. ${ }^{31}$ recently demonstrated that the introduction of $\mathrm{PbI}_{2}$ in HTL can suppress the aggregation of LiTFSI, thus improving device performance. TPFB doping potentially can also provide such advantages. In addition, TPFB itself has a very high solubility in CB solvents, so no extra polar solvent, which may damage the perovskite layer, is needed. With this motivation, we utilized TPFB to dope spiro-OMeTAD, and systematically investigated the effect of TPFB doping on this HTL and its influence on device performance. Thanks to the TPFB-doping strategy, we achieved dense and pinhole-free films with significantly increased conductivity and improved charge transport. Additionally, we found that TPFB doping improves device stability compared to conventional Li doping. Some other similar dopant molecules, such as triphenylborane (TPB) and 
tris(pentafluorophenyl)phosphine (TPFP), have also been explored. Our density functional theory (DFT) results revealed that the presence of the strongly electronegative fluorine atoms changes the electrostatic potential distribution in TPFB. Besides, they even make the benzene rings in TPFB as very strong Lewis acid sites, facilitating the formation of a Lewis acid-base adduct. Overall, our work demonstrates a facile and general doping method for the HTL leading to improved device efficiency and stability.

\section{Results and Discussion}

Figure 1a shows photographs of TPFB dopant, pristine spiro-OMeTAD, and TPFB-doped spiro-OMeTAD dissolved in $\mathrm{CB}$, respectively. As can be seen, the TPFB solution is highly transparent, whereas the pristine spiro-OMeTAD solution has a light yellow color. Upon TPFB addition to the spiro-OMeTAD solution, the resultant solution turns gradually red and dark gradually, which is an indicator for the formation of doped spiro-OMeTAD ${ }^{32}$. Despite that oxygen is not involved in the process, the electron transfer between spiro-OMeTAD and TPFB results in the oxidation of the spiro-OMeTAD molecule. We evaluated the optical absorption properties of TPFB-doped spiro-OMeTAD films along with undoped and Li-doped samples

(Figure 1b). The pristine sample shows almost no absorption near the $500 \mathrm{~nm}$ wavelength, and the corresponding absorption curve in the range is flat. Contrasting with this, a distinct broad absorption peak is present spanning from $440 \mathrm{~nm}$ to $550 \mathrm{~nm}$ after LiTFSI doping. The TPFBdoped sample shows a higher absorption than the Li-doped sample, possibly indicating stronger doping. Figure 1d shows the chemical structure of TPFB dopant molecules. The boron atom in TPFB is considered to have a strong electron-accepting capability and is a Lewis-acid site. The central nitrogen atom of the triphenylamine group in spiro-OMeTAD (Figure S1) has a lone electron pair (2s) and tends to donate electrons or share its electron pair. Therefore, spiroOMeTAD can form Lewis acid-base adducts with TPFB, where the effective charge-transfer process takes place. 
(a)

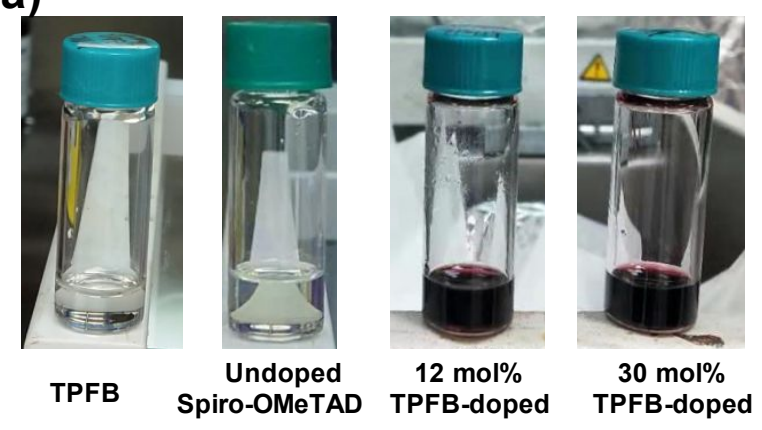

(c)

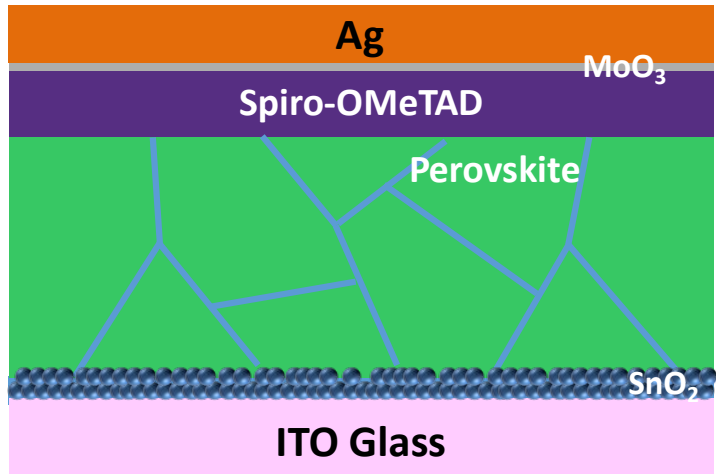

(b)

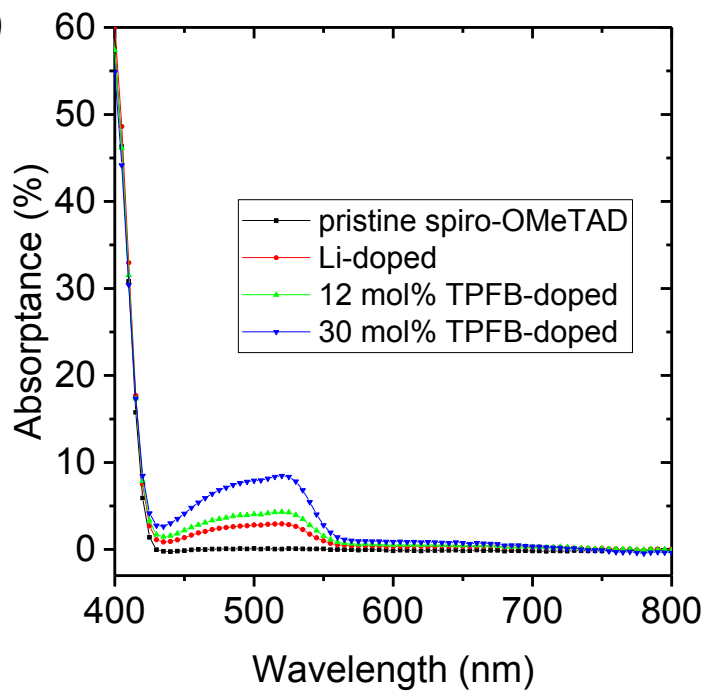

(d)

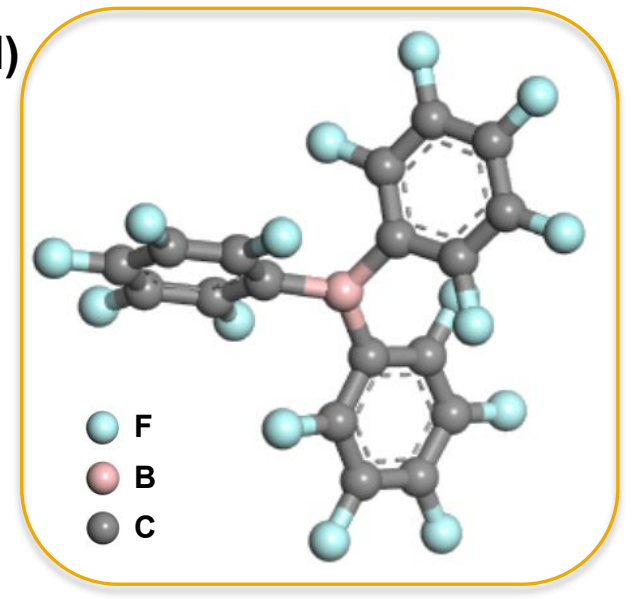

Figure 1. (a) Photographs of the dopant TPFB, undoped spiro-OMeTAD and TPFB-doped spiro-OMeTAD solution in chlorobenzene. (b) Absorptance spectra of the spin-coated spiroOMeTAD films. (c) The device structure of the PSCs in this study. (d) Chemical structure of the dopant tris(pentafluorophenyl)borane (TPFB).

Planar $n-i-p$ PSCs with a structure of ITO/ $\mathrm{SnO}_{2} /$ perovskite/spiro-OMeTAD/ $/ \mathrm{MoO}_{3} / \mathrm{Ag}$ with different spiro-OMeTAD doping concentrations were fabricated to reveal the impact of TPFB doping concentrations on the device performance. Note that we utilized mixed halide perovskite as an absorber layer for their high reproducibility and phase stability, and $\mathrm{SnO}_{2}$ as an electron-transport layer (ETL) to facilitate efficient charge transport at the ETL side $33-34$. Figure $\mathbf{S 2}$ shows the cross-sectional scanning electron microscopy (SEM) image of a complete device, which reveals the thickness of $\mathrm{SnO}_{2}$, perovskite, spiro-OMeTAD layer as $20 \mathrm{~nm}, 500$ $\mathrm{nm}$, and $70 \mathrm{~nm}$, respectively. For improved performance, we utilized $\sim 70 \mathrm{~nm}$ thick spiro- 
OMeTAD layers, which is significantly thinner than that used in some literature ${ }^{35-36}$. We prepared the control samples with the widely used LiTFSI/tBP dopants. Figure 2a shows the $J-V$ curves of the solar cells prepared with undoped, LiTFSI-doped and TPFB-doped spiroOMeTAD layers. Table S1 summarizes the photovoltaic parameters extracted from the champion $J-V$ measurements of the differently doped HTL devices. The Li-doped device exhibits the best $P C E$ of $19.1 \%$ with a $J_{s c}$ of $23.16 \mathrm{~mA} / \mathrm{cm}^{2}$, a $V_{o c}$ of $1.13 \mathrm{~V}$ and an $F F$ of $72.76 \%$. The device based on the undoped spiro-OMeTAD exhibits very poor performance, and the corresponding $J-V$ curve exhibits an S-shape, which is likely evidence of strong charge accumulation at the HTL/perovskite interface. On the other hand, with the optimum TPFB doping concentration of $12 \%$, The $J_{s c}, V_{o c}$ and $F F$ increased from $16.84 \mathrm{~mA} / \mathrm{cm}^{2}$ to 23.44 $\mathrm{mA} / \mathrm{cm}^{2}, 1.09 \mathrm{~V}$ to $1.14 \mathrm{~V}$, and $18 \%$ to $75 \%$, respectively. As a result, a maximum $P C E$ of $20.1 \%$ was obtained. The increased $F F$ and $J_{s c}$ can be explained by the increased charge-extraction capability and improved conductivity of the doped spiro-OMeTAD layer. However, excess TPFB in spiro-OMeTAD results in decreased device performance, especially in the $V_{o c}$ and $F F$, which can be assigned to increased impurity defects and decreased carrier mobility. The series resistance $\left(R_{S}\right)$ and the shunt resistances $\left(R_{s h}\right)$ were calculated using the 1/slope of the $J-V$ curves near $V_{o c}$ and $J_{s c}$ points, as shown in Table $\mathrm{S} 1$. The interface barrier, electrode resistance, and the internal resistance of each layer can contribute to $R_{S}$. We find that a high FF correlates with low $R_{s}$. $R_{s h}$ has also a significant effect on the shape of the $J$ - $V$ curve, and is usually related to the surface morphology between active layer and interfacial layers. In addition, intrinsic properties of the device such as charge transport and recombination can also influence $R_{s h}$. With TPFB doping and improved charge transport, high-performance devices also result in higher $R_{s h}$ values. For a better comparison, we show the statistical distribution of the device characteristics in Figure 2d and Figure S3. With comparison to the Li-doping, the $12 \mathrm{~mol} \%$ TPFB doping slightly improved $V_{o c}$ and $J_{s c}$, but the improvement in device performance mainly originates from $F F$. With the introduction of $45 \mathrm{~mol} \% \mathrm{TPFB}$, we observed a significant drop in $V_{o c}$ and $F F$ values. To analyze the hysteresis behavior of the devices, we performed forward/reverse scans for the $J-V$ measurements, which are shown in Figure S4. We found that the PCE values under reverse sweep condition are slightly higher than that under forward sweep condition. However, the hysteresis index $\left(1-P C E_{\text {forward }} / P C E_{\text {reverse }}\right)$ is still 
relatively small (less than 0.05 ), which could be attributed to good charge transfer properties of the $\mathrm{SnO}_{2}$ layer and the high quality of the perovskite layer ${ }^{37}$. To understand the effect of hysteresis, we determined the stabilized power output at a fixed voltage for the champion device, as shown in Figure 2b. Under a bias of $0.93 \mathrm{~V}$ near the maximum power point, the photocurrent could reach a stabilized value of $21.2 \mathrm{~mA} / \mathrm{cm}^{2}$, resulting in a stabilized PCE of 19.7\%. Figure 2d shows the external quantum efficiency $(E Q E)$ spectra for Li-doped and TPFB-doped devices. The integrated photocurrent density was found as 21.7 and $22.1 \mathrm{~mA} / \mathrm{cm}^{2}$, respectively, which are close to the $J_{s c}$ value achieved by $J-V$ measurements.

To show the universality of the TPFB doping for organic HTLs, we applied TPFB to other triphenylamine-based hole transport materials (HTMs) such as Poly[bis(4-phenyl)(2,4,6trimethylphenyl)amine (PTAA), 4,4'-Cyclohexylidenebis $\quad[N, \quad$ N-bis(4methylphenyl)benzenamine] (TAPC). We find that TPFB can be used to dope TAPC and PTAA as well which can be evidenced by the device results shown in Figure S6. As can be seen, the undoped TAPC-based device exhibits a very low performance. After TPFB doping in TAPC, the device performance was significantly improved and did not change much within a large doping range. Compared with spiro-OMeTAD devices, the doped TAPC-based devices still exhibit a lower $F F$, which may be due to the difference in energy level matching and low mobility. Nonetheless, this indicates TPFB dopant is universal for doping of triphenylaminebased HTM. 

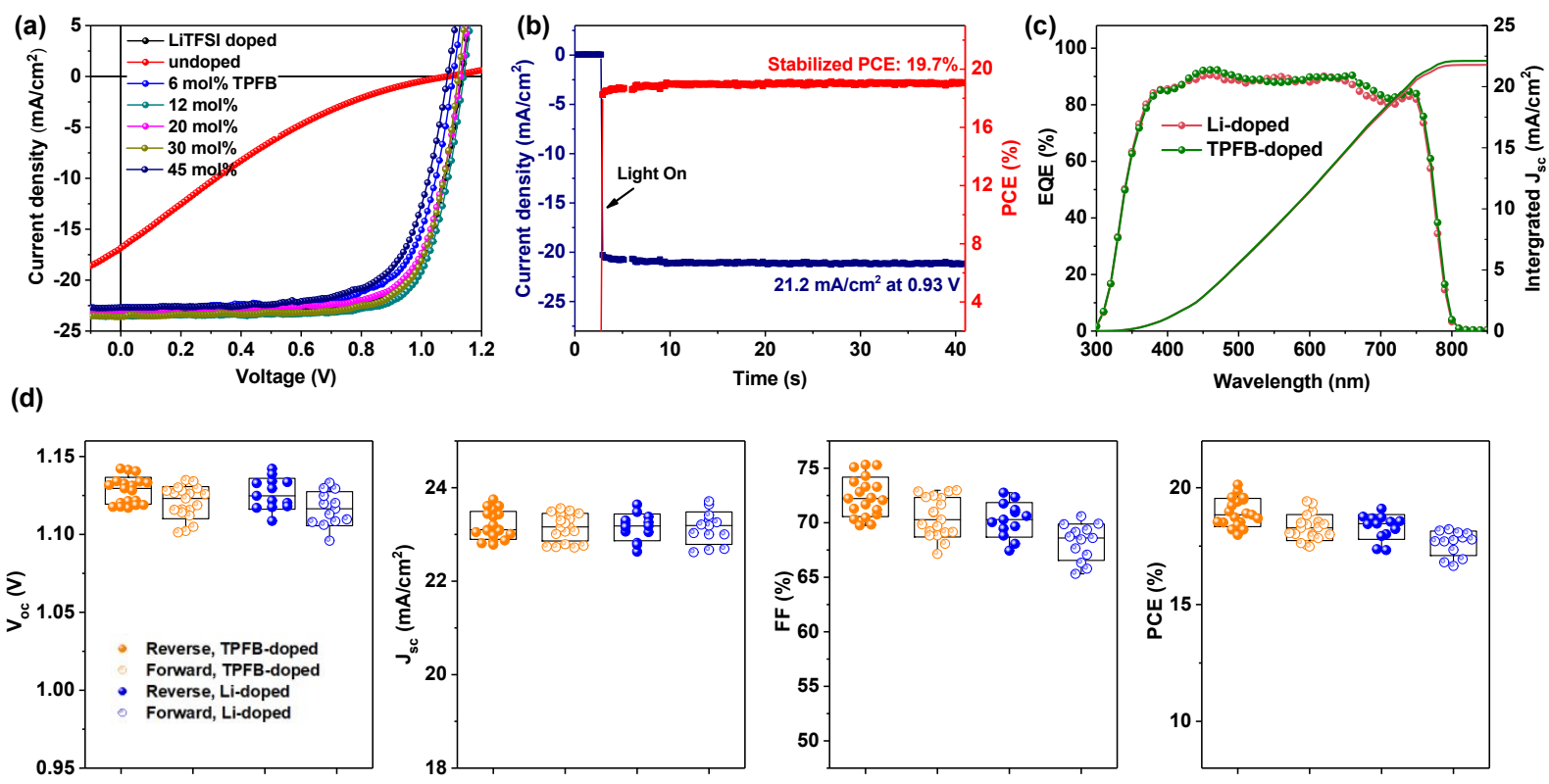

Figure 2. (a) J-V curves of the perovskite solar cell fabricated with Li-doped, undoped, and TPFB-doped spiro-OMeTAD layer. (b) Photocurrent density and stabilized power output measured at near maximum power point for the champion device with the TPFB doping. (c) IPCE spectra of the corresponding perovskite solar cells. (d) The statistical distribution of the performance parameters for the devices with Li-doped and 12 mol\% TPFB-doped spiroOMeTAD layers.

We used Kelvin probe force microscopy (KPFM) to measure the contact potential difference $\left(\mathrm{CPD}, V_{\mathrm{CPD}}\right)$ between a conducting tip and the samples. Using highly ordered pyrolytic graphite (HOPG) with a known work function $(W F)$ of $4.6 \mathrm{eV}$ as a calibration sample ${ }^{38}$, we obtained the absolute $W F$ of the spiro-OMeTAD layers before and after doping, as shown in Figure 3a. The measured KPFM potential or $V_{\mathrm{CPD}}$ values correspond to the average values of the acquired KPFM images. The $W F$ of the sample is defined as $\Phi_{\text {sample }}=\left(V_{H O P G}-V_{\text {sample }}\right)^{*} e+\Phi_{H O P G}$, where $\Phi_{H O P G}$ is the work function of HOPG, $V_{H O P G}$ and $V_{\text {sample }}$ are the measured KPFM potentials of the HOPG and the sample, and $e$ is the electronic charge. The mean KPFM potential is 491, 12 and $-174 \mathrm{mV}$ for the pristine, Li-doped, and TPFB-doped spiro-OMeTAD, giving corresponding $W F$ of $4.18,4.69$ and $4.85 \mathrm{eV}$, respectively. A slight increase in $W F$ was observed after 24 hours of exposure to air for the pristine and Li-doped samples. In contrast, 
there is almost no change in the $W F$ of the TPFB-doped sample. Even after oxidation in air, the pristine sample still has a low $W F$ of $4.29 \mathrm{eV}$, which inevitably causes a barrier for hole transport from the perovskite to the HTL. The Li-doped sample after 24 hours of air exposure and the TPFB-doped sample showed a work function of 4.87 and $4.84 \mathrm{eV}$, which is close to the valence band maximum ( $V B M)$ of the perovskite layer, thereby effectively avoiding hole accumulation.

Figure 3b shows the $I-V$ characteristics of the spiro-OMeTAD layer, acquired from the socalled hole-only device structure of ITO/PTEOT:PSS/spiro-OMeTAD/MoO $/ \mathrm{Mg}_{3} / \mathrm{As}$ can be seen, the current improved obviously after Li or TPFB doping. For the pristine sample, the current at $0.1 \mathrm{~V}$ is around $10^{-5} \mathrm{~A}$. Upon Li and TPFB doping, the current increased by one and two orders of magnitude, respectively. For the pristine sample, the $I-V$ curve exhibits more or less space-charge-limited-current (SCLC) behavior with a trap-filled limit voltage $\left(V_{\mathrm{TFL}}\right)$ of $0.425 \mathrm{~V}$. After the doping, the SCLC characteristic becomes less clear, which is attributed to an obviously increased carrier density in the spiro-OMeTAD bulk. Especially after the TPFB doping, the $I-V$ curve is almost linear in the voltage range of 0 to $2 \mathrm{~V}$. Within that range, the current is primarily consisting of contributions from the free carriers of the doped sample rather than charge traps. Based on the linear portion of the $I-V$ characteristic and subtracting the resistance of the sample without spiro-OMeTAD, we can estimate the conductivity of the spiroOMeTAD layer, which is $3.4 \times 10^{-2} \mu \mathrm{S} / \mathrm{cm}, 0.47 \mu \mathrm{S} / \mathrm{cm}$, and $9.1 \mu \mathrm{S} / \mathrm{cm}$ for the undoped, Lidoped and TPFB-doped samples, respectively.

We used ultraviolet photoelectron spectroscopy (UPS) to further investigate the change in the electronic properties of the spiro-MeOTAD film before and after doping. Figure 3c, d show the photoemission cutoff and onset region of the spiro-OMeTAD layer, which can be used to obtain results on $W F$ and highest occupied molecular orbital (HOMO) position (relative to the Fermi level $E_{\mathrm{F}}$ ), respectively. The band diagrams of the spiro-OMeTAD layers are summarized in Figure 3e. The work function of the undoped spiro-OMeTAD film is $4.32 \mathrm{eV}$, while those of the Li-doped and TPFB-doped films are 4.62 and $4.72 \mathrm{eV}$, respectively; these values correspond well with those obtained from our KPFM measurements above. The HOMO position of the undoped film is at $0.73 \mathrm{eV}$ below $E_{\mathrm{F}}$. By combining $W F$ and HOMO position, the ionization energy (IE) could be calculated, which is $5.05 \mathrm{eV}$ for the undoped film. The 
change in $I E$ after Li and TPFB doping is very small, ranging from 5.05 to $5.11 \mathrm{eV}$, in accord with some previous reports ${ }^{20,39}$. In contrast, the $E_{\mathrm{F}}$ shift downward obviously when spiroOMeTAD were doped with Li salt and TPFB. Overall, these results are consistent with the KPFM and conductivity measurement results. The Fermi level exhibits a larger downward shift under $12 \mathrm{~mol} \%$ of TPFB doping than that under Li doping. Further increasing the doping concentration of TPFB to $30 \mathrm{~mol} \%$ does not seem to cause further changes in energy levels. This may indicate that $12 \mathrm{~mol} \%$ of the TPFB dopant is sufficient for doping of the spiroOMeTAD film.

Li et al. reported that some inorganic acids such as acetic acid, sulfuric acid, and phosphoric acid could not alone effectively dope spiro-OMeTAD. ${ }^{32}$ However, in this study, the spiroOMeTAD layer after doping with Lewis acid TPFB shows an obvious improvement in conductivity, which is $\sim 260$ fold higher than that of undoped one. This is because the role of the Lewis acid in the doping of spiro-OMeTAD layer is completely different from that of inorganic acids. The inorganic acid molecules themselves are not able to be complexed with spiro-OMeTAD, but the acids can release hydrogen ions and rely on hydrogen bonding to promote the oxidation of spiro-OMeTAD in the presence of TFSI-based salts $^{32}$. The predominant role played in LiTFSI doping comes from TFSI, which can form stable chargetransfer complexes with spiro-OMeTAD when exposed to air. This is further supported by the fact that many Li-free TFSI-containing additives have been shown to successfully dope spiroOMeTAD layer ${ }^{40-41}$. However, the doping of the Lewis acid TPFB depends on its ability to form a Lewis adduct. TPFB has a strong complexing capability; it can combine with spiroOMeTAD to form a Lewis spiro-OMeTAD-TPFB adduct, which results in electron delocalization and leaves free charge in the spiro-OMeTAD matrix, thereby increasing conductivity. In a broad sense, LiTFSI doping can also be considered as a Lewis acid-base reaction via formation of spiro-OMeTAD ${ }^{+} \mathrm{TFSI}^{-}$. 


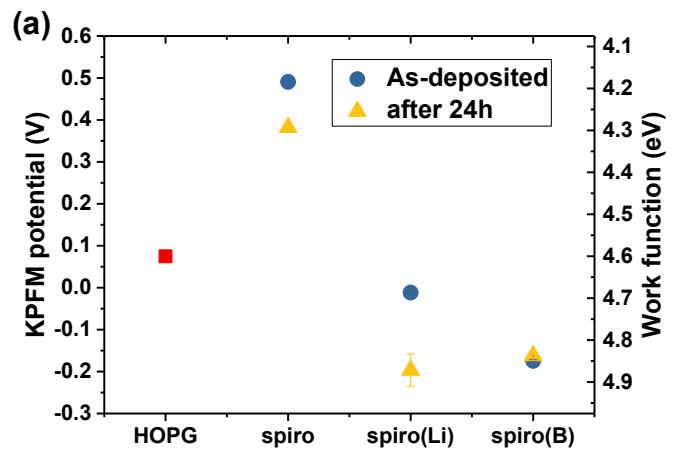

(b)
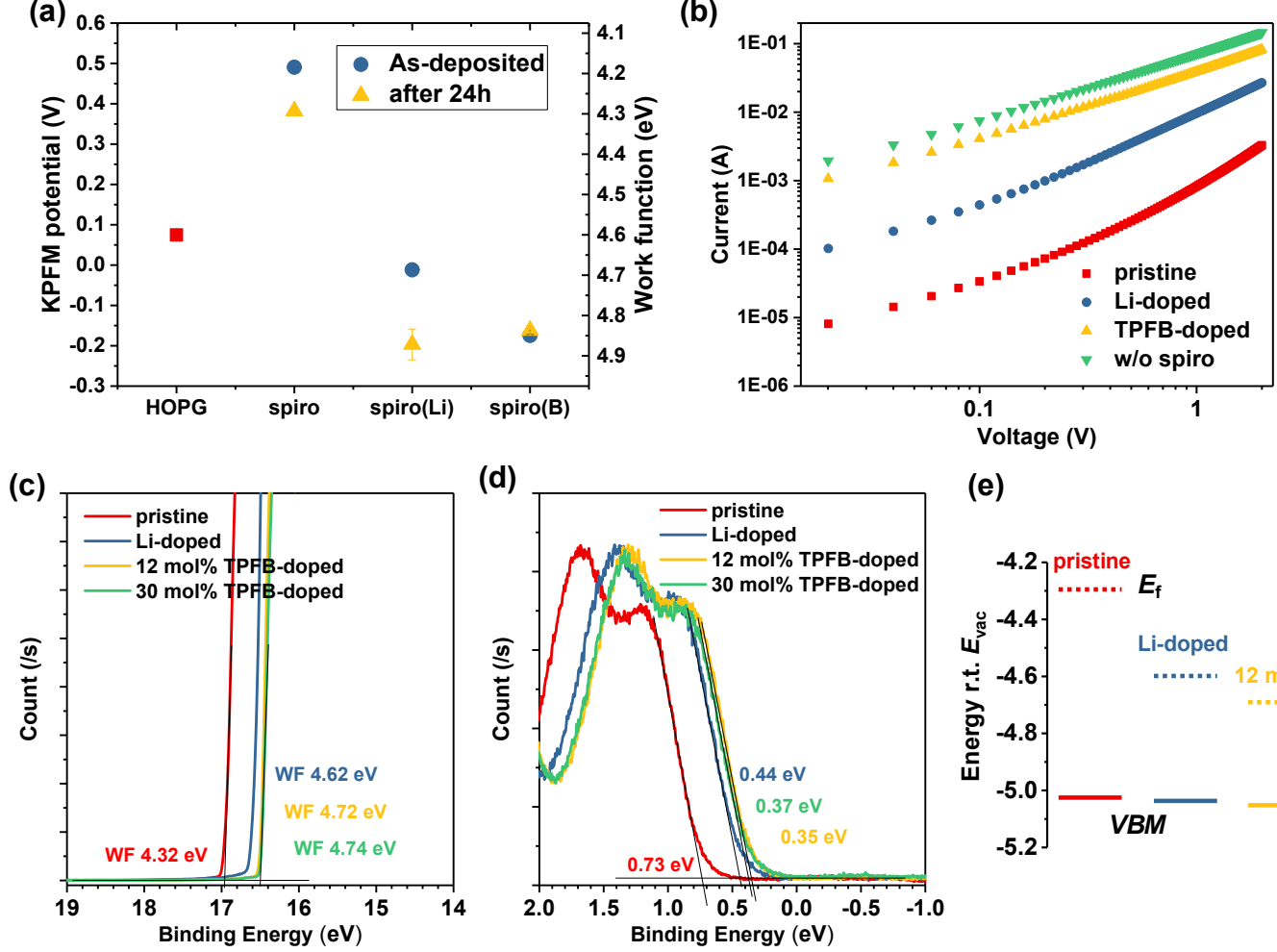

(d)

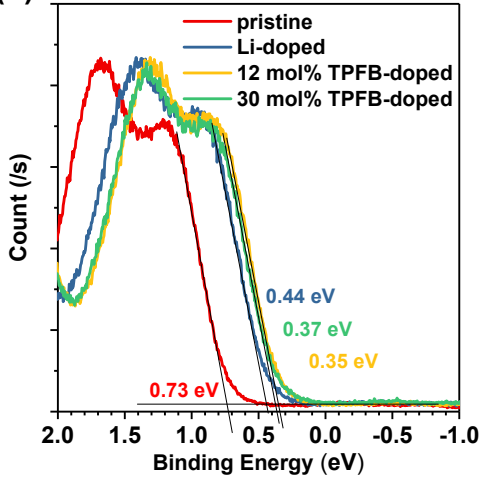

(e)

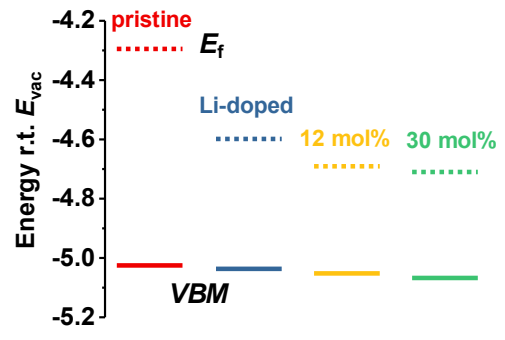

Figure 3. (a) KPFM surface potential of and calculated work function of undoped, Li-doped, and TPFB-doped spiro-OMeTAD layer, together with HOPG reference samples. (b) I-V characteristics of the spiro-OMeTAD layer with the device structure ITO/PEDOT:PSS/spiro$\mathrm{OMeTAD} / \mathrm{MoO}_{3} / \mathrm{Ag}$. (c) Photoemission cutoff spectra and (d) valence band structure of the spiro-OMeTAD layer, determined by ultraviolet photoelectron spectroscopy (UPS). (e) Energy level diagrams extracted from UPS spectra.

In order to obtain the charge extraction characteristics, we measured steady-state and transient PL spectra for the perovskite films coated without and with the different spiroOMeTAD layers, as shown in Figure 4a and $\mathbf{4 b}$, respectively. Here, all the perovskite films were prepared under the same conditions. For the steady-state PL analysis, using perovskite/HTL stack, the quenching of the PL intensity is generally assumed as a measure of the efficient charge extraction. When spiro-OMeTAD layer was coated onto the perovskite surface, the steady-state PL intensity was reduced to one-thirtieth of the uncoated one, and the average PL decay time is reduced from 284 ns to $60 \mathrm{~ns}$ (see Table S2), which is consistent with the function of the spiro-OMeTAD layer as a charge extraction layer. The sample with TPFB- 
doped spiro-OMeTAD exhibits the lowest PL intensity, which indicates the dopant TPFB itself can not act as a charge transport layer in perovskite solar cells, and TBFB doping does not reduce the quenching effect of spiro-OMeTAD. A slight improvement in PL intensity and decay lifetime was observed when the perovskite layer was coated by only TPFB, which may be related to additional passivation effect of Lewis acid on the perovskite surface ${ }^{42-45}$.

Transient photocurrent under zero bias and transient photovoltage under open-circuit conditions were used to characterize the charge-extraction and charge-recombination lifetime, as shown in Figure 4c and Figure S7. The charge-recombination lifetime of the TPFB-doped device was $43.1 \mu \mathrm{s}$, compared to $35.1 \mu \mathrm{s}$ for the Li-doped device. The charge-extraction time measured at zero bias is $1.52 \mu$ s for the TPFB-doped device, compared with $2.15 \mu$ s for the Lidoped device. A faster extraction time implies weaker recombination, which is associated with longer charge-recombination lifetime. We also obtained the charge recombination lifetime using impedance analysis. The extracted recombination resistance $R_{\text {rec }}$ and chargerecombination lifetime $\tau_{r-i s}$ are shown in Figure S9. A higher $R_{\text {rec }}$, which is inversely related to the recombination rate, and longer $\tau_{r-i s}$ for TPFB-doped device compared with the Li-doped device was observed, which is also consistent with the TPV measurement. These findings indicate a better charge transfer and lower interface recombination in the TPFB-doped devices.

We measured the devices under different illumination intensities, and derived the $V_{o c}$ and $J_{s c}$, as shown in Figure 4d, e. The detail of $J-V$ curves can be seen in Figure S10. The opencircuit voltage $V_{o c}$ can be approximated as $V_{o c}=\frac{n k T}{q} \ln \frac{J_{s c}}{J_{o}}$, where $n$ is the diode quality factor. In the normal diode case, where the superposition principle holds, $J_{s c}$ scales linearly with the light intensity. Thus, from the semi-logarithmic plot of the $V_{o c} v s$. light intensity, we can find a linear relationship, with the slope following the expression slope $=\frac{n K T}{q} \log _{10} e$. By linear fitting, we calculated an $n$ value of 1.32 for the Li-doped device, 1.25 for the TPFB-doped device, and 1.81 for the undoped device. The $n$ value is associated with the recombination mechanism of the device. Improvement in device performance is accompanied by a decrease in $n$ value. Many detailed studies have established now that further improvements in perovskite devices rely primarily on the mitigation of nonradiative recombination, particularly at the interface. In the case of TPFB doping, the device shows an $n$ value close to 1, indicating a very low 
recombination loss. Thus, we can correlate the performance improvement of TPFB doped devices to a reduction in recombination. In Figure 4e, the relationship between $J_{s c}$ and light intensity is almost linear for the Li-doped and TPFB-doped cases. In contrast, for the pristine device, $J_{s c}$ is very low at high light intensity conditions, which is limited by the high series resistance of the undoped HTL.

To get insight into the built-in potential and depletion region of the device, we applied MottSchottky analysis by measuring the capacitance-voltage $(\mathrm{C}-\mathrm{V})$ characteristics of the complete devices under dark conditions (Figure 4f). The relevant equation is given by $\frac{1}{C^{2}}=\frac{2\left(V_{b i}-V\right)}{\varepsilon \varepsilon_{0} q N A^{2}}$, where $C$ is the measured capacitance, $A$ is the device area, $\varepsilon_{0}$ is the vacuum dielectric constant, $\varepsilon$ is the relative dielectric constant of the active layer (35.7 of $\varepsilon$ value was obtained from Figure S11), $q$ is the elementary charge, $V_{b i}$ is the built-in potential, $V$ is the applied voltage. MottSchottky plots show the cutoff voltage at $1.05 \mathrm{~V}$, which represents the built-in potential of the devices. This indicates that the TPFB-doped device shows almost the same flat-band potential as the Li-doped device. The capacitance exhibits a broad plateau in voltage of -0.2 to $0.5 \mathrm{~V}$, which can be attributed to the perovskite film layer as the intrinsic layer in the $n-i-p$ device configuration. The capacitance exhibits a drop only when the applied voltage approaches the flat band potential regime. The depletion width $\left(W_{d}\right)$ can be calculated using the equation $W_{d}=$ $\varepsilon \varepsilon_{0} A / C$, as shown in Figure S12. The calculated $W_{d}$ value is comparable to the thickness of the perovskite layer. 
(a)

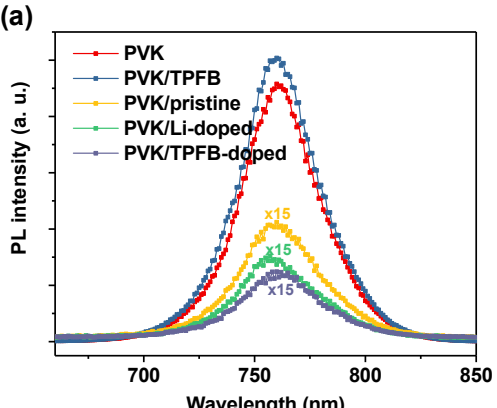

(d)

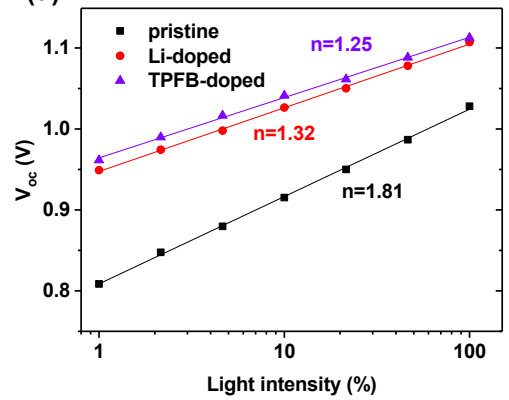

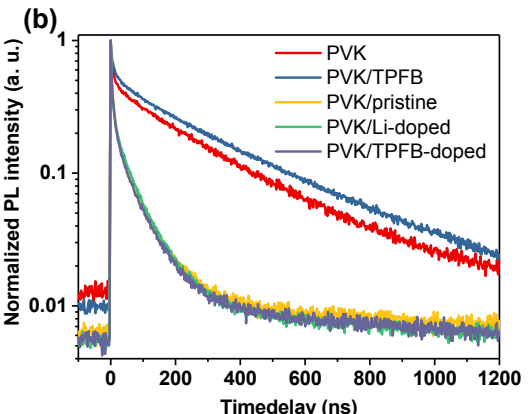

(e)

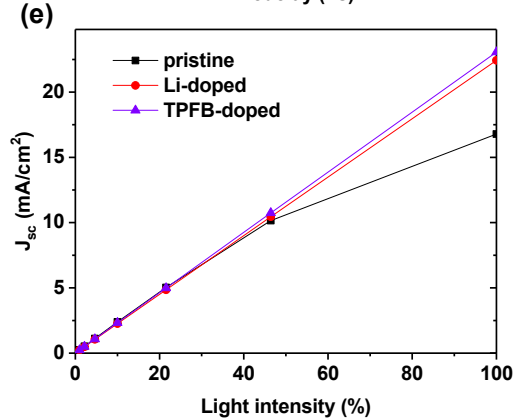

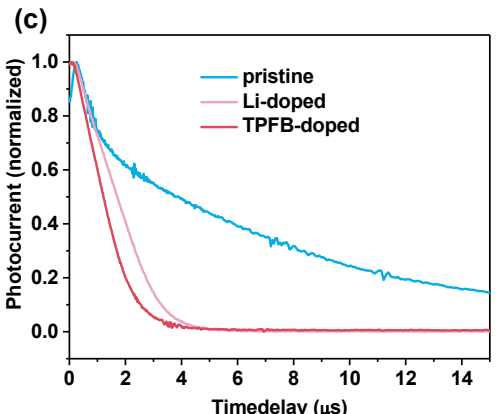

(f)

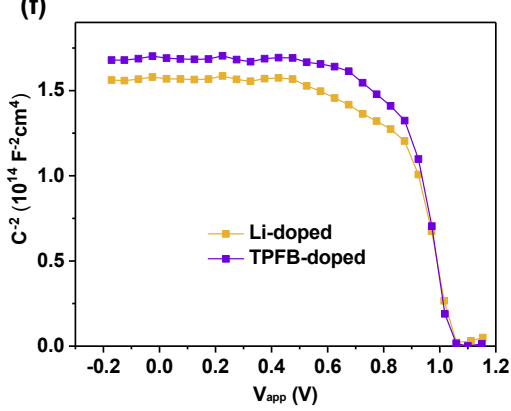

Figure 4. (a) Steady PL and (b) TRPL spectra of the bare perovskite film and perovskite films coated with TPFB dopant, undoped, Li-doped, and TPFB-doped spiro-OMeTAD layer. (c) Transient photocurrent decay of the perovskite devices. The dependency of the (d) $V_{o c}$, (e) $J_{s c}$ of the perovskite devices prepared with undoped, Li-doped, and TPFB-doped spiro-OMeTAD layers. (f) Mott-Schottky plots for perovskite solar cells.

To examine the effect of the TPFB doping on the device stability, we performed long-term stability tests of the devices. The un-encapsulated devices were kept at room temperature in an ambient environment where the relative humidity monitored by a hygrometer was typically about $20-35 \%$. The devices were taken out from time to time for $J-V$ measurements. The air stability test results are shown in Figure 5a and Figure S13. In all cases, the change in $V_{o c}$ and $J_{s c}$ is relatively small over $1100 \mathrm{~h}$ of storage time, the $V_{o c}$ of some cells even improves a little in the initial 100 hours. As time goes by, $J_{s c}$ gradually declines. The main difference in the stability results is the change in $F F$. The degradation of PSCs commonly is first initiated at the interface or the grain boundaries, which will increase the resistance of charge transport, thereby reducing the $F F$. The evolution of the normalized $F F$ shows that TPFB-doped devices exhibit a significantly improved long-term stability, compared to Li-doped device. Therefore, the TPFB doped devices retained near $85 \%$ of their original PCE, while for the TPFB-doped device this is only $75 \%$. In order to find possible underlying reasons, we performed atomic force 
microscopy (AFM) analysis on the Li-doped and TPFB-doped films, which is shown in Figure 5d, e. There are always many pinholes in the case of the Li-doped spiro-OMeTAD layer, whereas the TPFB-doped film is smooth and pinhole-free. This structural difference led us to think that the presence of pinholes in the Li-doped film may provide pathways for humidity to permeate into the perovskite layer, thus accelerating the degradation. Previous studies revealed that these pinholes are associated with tBP evaporation and Li salt aggregation ${ }^{21}$. Water contact angles of the spiro-OMeTAD films with LITFSI and TPFB dopants were investigated to understand the impact of the dopants on the wettability behavior of spiro-OMeTAD, as shown in Figure 5f. The un-doped and TPFB-doped films have high water contact angles of $78.7^{\circ}$ and $78.6^{\circ}$, respectively. With TPFB doping, the contact angles remain almost the same and might offer an advantage in the long-term stability of the devices. Nonetheless, once the spiroOMeTAD film is doped with Li salt, its hydrophobicity is reduced considerably which can be evidenced by the reduced contact angle to $71.9^{\circ}$. In addition to the function of charge transport, the spiro-OMeTAD layer can also serve as a protective layer to inhibit the absorption of water molecules by the perovskite layer. Therefore, we can speculate that Li-doping increases the tendency towards moisture absorption and perovskite degradation. Obviously, the TPFB-doped layer is more hydrophobic compared with the Li-doped layer, which can provide better protection for the perovskite layer in a humid environment. We also examined the thermal stability of the perovskite devices at $85^{\circ} \mathrm{C}$, as shown in Figure 5b. Although the TPFB-doped devices showed improved thermal stability compared with the Li-doped devices, both of the devices degraded quickly in $190 \mathrm{~h}$. This seems to be a common problem with the spiroOMeTAD-based $n-i-p$ type perovskite device and may be resolved in future study. One important reason for the poor thermal stability is that spiro-OMeTAD exhibits a very poor barrier capability at elevated temperature; the $\mathrm{MoO}_{3} / \mathrm{Ag}$ electrode layer can then easily diffuse across spiro-OMeTAD layer, thereby damaging the perovskite layer and causing fast degradation $^{46-47}$. 

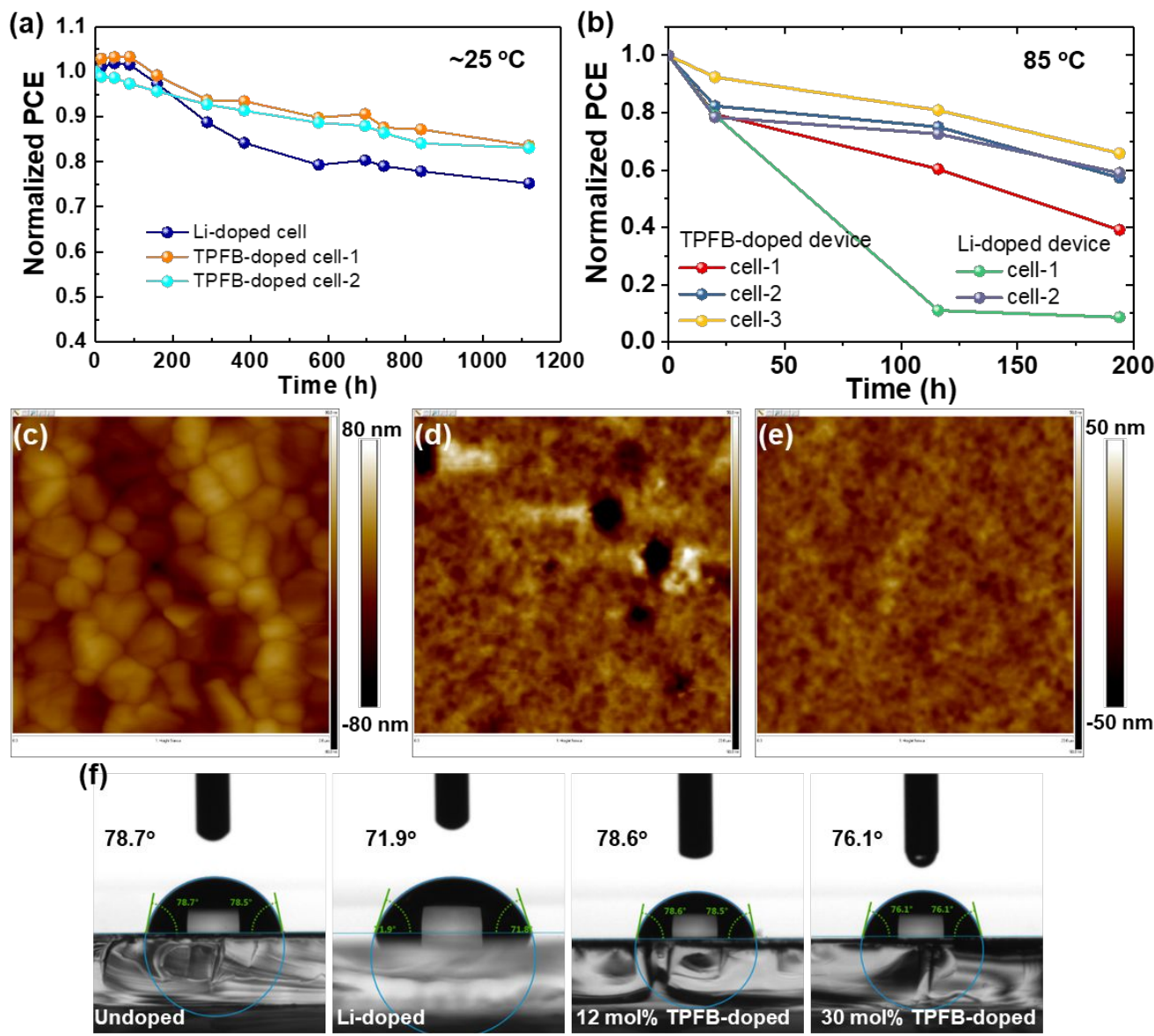

Figure 5. (a) Long-term air stability of the un-encapsulated solar cells stored at room temperature at a relative humidity of about $20 \%-35 \%$. (b) Thermal stability of the TPFB-doped and Li-doped devices. The devices were placed at $85^{\circ} \mathrm{C}$ on a hot plate under $\mathrm{N}_{2}$ atmosphere. AFM height images of the (c) bare perovskite film, the films coated with (d) Li-doped and (e) TPFB-doped spiro-OMeTAD layer. (f) Water contact angle on spiro-OMeTAD films.

In order to shed light on the role of fluorine and boron atoms, we examined the effect of TPB and TPFP materials as a dopant in spiro-OMeTAD. The TPB has a similar structure to TPFB but has no fluorine atoms, and the central site in TPFP is a phosphorus atom instead of a boron atom. The corresponding device results are shown in Figure 6a. The device with TPB dopant exhibits very low $F F$ values and a strong S-shape feature, which is similar to that with undoped spiro-OMeTAD. This indicates that spiro-OMeTAD can hardly be doped with TPB, although TPB is also a kind of Lewis acid, highlighting the importance of fluorination in TPFB. Interestingly, the spiro-OMeTAD layer seems to be doped with TPFP, but the doping is 
insufficient, and the obtained $F F$ for the TPFP-doped device is only about $60 \%$. Visual inspection of the solutions (Figure 6b) also gives some information as the color change is associated with oxidation of spiro-OMeTAD. When TPB was added, the color of the solution did not change, but when TPFB was added, the color became a little darker.

To further understand the fluorination effect on the molecular configurations and electronic properties of TPFB, DFT simulations were carried out using the DMol3 module in Materials Studio. The electrostatic potential, HOMO and lowest unoccupied molecular orbital (LUMO) level are shown in Figure 6c and Figure S16. For comparative purposes, calculations on fluorine-free TPB and boron-free TPFP material were also performed. As can be seen, the maximum local electrostatic potential is located at the center of the molecules. This means that the central moiety on the molecules is a strong Lewis acid site, which tends to pair electrons with the Lewis base HTM. In addition, for fluorine-containing TPFB and TPFP molecules, due to the addition of fluorine atoms, the local electrostatic potential near the benzene rings becomes very strong, which makes the benzene rings as Lewis acid site with a large crosssectional area, thereby promoting the formation of Lewis acids and bases. This explains why fluorine-containing TPFB and TPFP can dope spiro-OMeTAD better than TPB. The HOMO and LUMO levels (Figure S16) of TPB, TPFP and TPFB were determined to be $-5.91 /-2.45$ $\mathrm{eV},-6.46 /-2.91 \mathrm{eV}$ and $-6.64 /-4.04 \mathrm{eV}$, respectively. P-type doping would be facilitated by charge transfer from the HOMO level of the organic matrix to the LUMO of the dopant molecule ${ }^{8}$. The fluorine-containing TPFB material has the lowest LUMO value among these three materials, which matches best with the HOMO of spiro-OMeTAD. This implies that electron transfer is most likely to occur between TPFB and spiro-OMeTAD. 

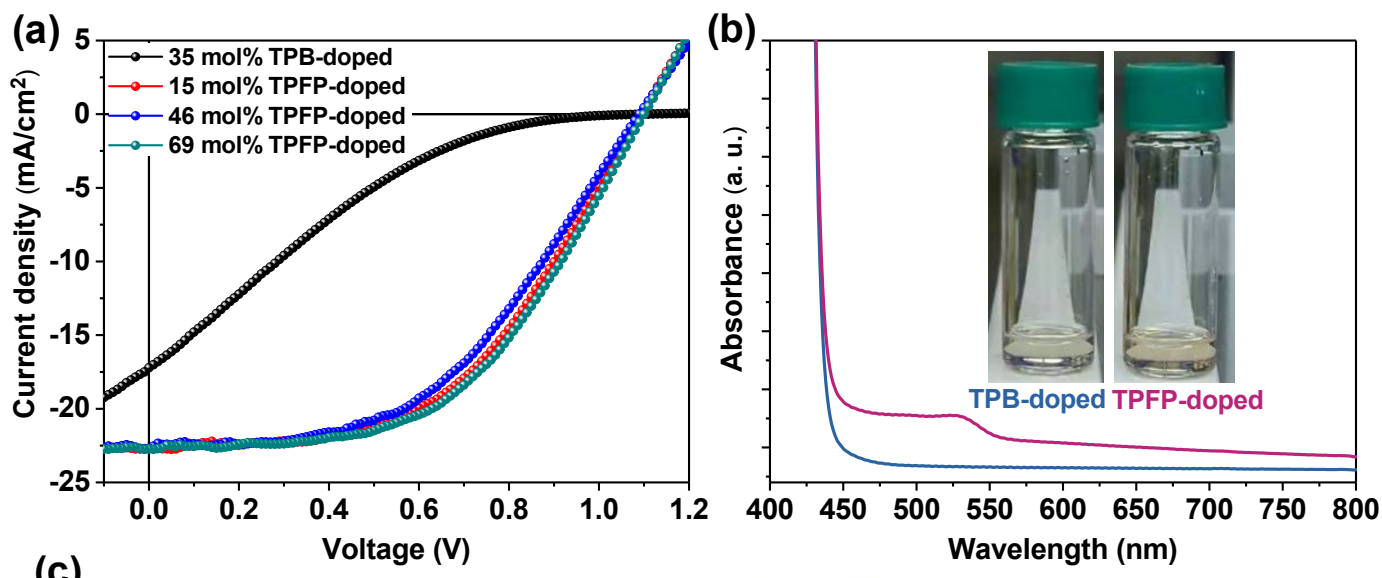

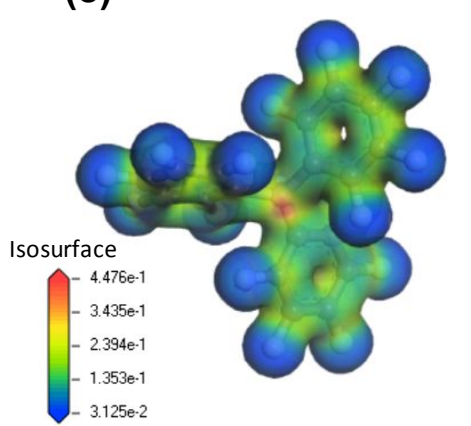

TPFB

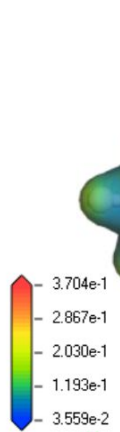

- $3.559 \mathrm{e}-2$

\section{Conclusions}

In summary, we show that TPFB can be used to effectively dope organic hole transport materials, increase their conductivity, and thus improve the corresponding device performance. Such performance improvement has been observed in the cases of spiro-OMeTAD and other triphenylamine-based materials, demonstrating the general applicability of this doping method. This dopant induces a larger downward shift of Fermi level and reduces recombination loss, leading to a higher $J_{s c}$ and $F F$ compared to the Li-doped device. Moreover, the TPFB-doped devices show significantly improved long-term stability. Theoretical calculations indicate that the central boron atom in TPFB is a strong Lewis acid site. More critically, due to the strong 
electronegativity of the fluorine atoms on the side of the benzene rings, the entire benzene rings can become a large Lewis acid site, facilitating the formation of Lewis acid-base adduct. The fundamental understanding from the Lewis acid-base interaction point of view may provide new insights and opportunities for developing new HTM dopants.

\section{Experimental Section}

\section{Solar cell fabrication:}

Before the deposition of other films, the ITO glasses were ultrasonically cleaned with detergent, deionized water, acetone, and isopropanol successively, and then blow-dried in nitrogen. A $\mathrm{SnO}_{2}$ nanocrystal solution was prepared by diluting 1 part of the $\mathrm{SnO}_{2}$ colloidal solution (Alfa Aesar, $15 \%$ in $\mathrm{H}_{2} \mathrm{O}$ colloidal dispersion) into 3 parts of deionized water and 3 parts of isopropanol (1:3:3 volume ratio). The diluted $\mathrm{SnO}_{2}$ solution was spin-coated on glass/ITO substrates at $3000 \mathrm{rpm}$ for $50 \mathrm{~s}$, followed by drying at $150{ }^{\circ} \mathrm{C}$ for $20 \mathrm{~min} .1 .2 \mathrm{M}$ $\mathrm{Cs}_{0.05} \mathrm{FA}_{0.80} \mathrm{MA}_{0.15} \mathrm{PbI}_{2.55} \mathrm{Br}_{0.45}$ perovskite precursor solution with excess lead iodide was prepared by dissolving FAI (Greatcell Solar), MABr (Greatcell Solar), CsI (Sigma Aldrich), $\mathrm{PbI}_{2}$ (Alfa Aesar), and $\mathrm{PbBr}_{2}$ (Alfa Aesar) in a mixed solvent (DMF/DMSO=4:1). $70 \mu \mathrm{L}$ of perovskite solution was spun onto the substrates at $2000 \mathrm{rpm}$ for $10 \mathrm{~s}$ and $4000 \mathrm{rpm}$ for $30 \mathrm{~s}$. $150 \mu \mathrm{L}$ of chlorobenzene was dropped in the center of the substrates $10 \mathrm{~s}$ before the end of the spin-coating process. After the rotation ceased, the substrates were immediately transferred onto a hotplate of $100{ }^{\circ} \mathrm{C}$ and annealed for 30 mins. The pristine spiro-OMeTAD solution was prepared by dissolving $36 \mathrm{mg}$ spiro-OMeTAD (Lumtec) in $1 \mathrm{~mL}$ anhydrous chlorobenzene (Sigma-Aldrich). The tris(pentafluorophenyl)borane (TPFB) doped spiro-OMeTAD solutions were prepared by mixing TPFB with the pristine solution with varied ratios. For Li-doped spiro-OMeTAD solution, $15 \mu \mathrm{L}$ of 4-tert-butylpyridine, $9 \mu \mathrm{L}$ of lithium bis(trifluoromethylsulfonyl)imide (LiTFSI) solution $(520 \mathrm{mg} / \mathrm{mL}$ in acetonitrile) was added into $1 \mathrm{~mL}$ pristine solution. Spiro-OMeTAD films were obtained by spin coating at $3000 \mathrm{rpm}$ for $30 \mathrm{~s}$. The devices were finished by thermally evaporating $10 \mathrm{~nm} \mathrm{MoO}_{3}$ and $100 \mathrm{~nm}$ silver successively. 


\section{Characterization:}

All devices were tested using Keithley 2400 at room temperature under AM 1.5G illuminations $\left(1000 \mathrm{~W} / \mathrm{m}^{2}\right)$ from an Abet Technologies Sun 3000 solar simulator which was calibrated using a standard silicon cell (RERA). Current-voltage (J-V) curves were obtained both in reverse $(1.2 \mathrm{~V} \rightarrow-0.1 \mathrm{~V})$ and forward scan $(-0.1 \mathrm{~V} \rightarrow 1.2 \mathrm{~V})$ with a step size of $10 \mathrm{mV}$. Absorptance spectra were obtained using a PerkinElmer Lambda 950 UV-vis-NIR spectrophotometer with an integrating sphere. The external quantum efficiency (EQE) of the solar cell was measured with a commercial EQE system (Newport). The chopped monochromatic light beam was focused entirely on the active area of the solar cells. SEM images were obtained with a ZEISS Auriga Crossbeam system with an accelerating voltage of $5 \mathrm{kV}$. Steady photoluminescence (PL) and time-resolved photoluminescence (TRPL) measurements were carried out with a spectrofluorometer (Fluoroma-4, Horiba Scientific). For steady PL, $500 \mathrm{~nm}$ monochromatic light was continuously illuminated on the surface of the films. For TRPL, a $633 \mathrm{~nm}$ pulsed laser was used for the pump excitation and $760 \mathrm{~nm}$ light was probed. Atomic force microscopy (AFM) images were obtained with tapping mode using a Digital Instruments Multimode AFM (Veeco Metrology Group). For Kelvin probe force microscopy (KPFM) measurement, a NT-MDT AFM system (Solver) equipped with a Pt coated Si cantilever (OSCM-PT, Veeco) was used. A high ordered pyrolytic graphite (HOPG) was used as a calibration sample. The work function and valence band edge of the films was obtained by ultraviolet photoelectron spectroscopy (UPS) using an Omicron-UHV-System equipped with a He lamp. Transient photocurrent (TPC), transient photovoltage (TPV), capacitance and impedance measurements were carried out in a glove box using the commercial Paios system (FLUXiM, Winterthur, Switzerland). Specifically, impedance spectra (IS) were obtained with a $20 \mathrm{mV}$ rms amplitude over the frequency range of $300 \mathrm{~Hz}$ to $1 \mathrm{MHz}$ under a LED illumination equivalent of 1 sun. Capacitance-voltage (C-V) measurements were carried out in dark at $1 \mathrm{kHz}$ with applied potential between -0.2 and $1.2 \mathrm{~V}$. For TPV measurement, an LED pulse light with a pulse length of $1 \mu$ s plus a constant bias light was used to modulate the $\mathrm{V}_{\mathrm{oc}}$. The contact angle was measured by the KRUSS drop shape analyzer.

Density functional theory (DFT) was performed using the program package DMol3 in 
Materials Studio 2017 R2 (Accel-rys Inc.). The exchange-correlation function that was employed was the generalized-gradient approximation, Perdew-Burke-Ernzerhof (GGA-PBE) functional. The double-numeric quality basis set with polarization functions (DNP) was used. Orbital cutoff was set at $3.7 \AA$. In the geometry optimization calculations, the convergence tolerances of energy, maximum force, and maximum displacement were $2 \times 10^{-5} \mathrm{Ha}$ (1 $\mathrm{Ha}=27.2114 \mathrm{eV}), 0.004 \mathrm{Ha} / \AA$, and $0.005 \AA$, respectively.

\title{
Supporting Information
}

Chemical structures; SEM images; statistics of device parameters; solution photos; J-V curves; TRPL lifetime; transient photovoltage; Nyquist plots; impedance spectroscopy; J-V curves with different light internsity; CELIV measurement; depletion region widths; air stability test; optical micrograph; thermal stability test; ball and stick model; DFT calculation

\author{
AUTHOR INFORMATION \\ Corresponding Authors \\ *Email: jiang.liu@kaust.edu.sa (J.L.). \\ *E-mail: stefaan.dewolf@kaust.edu (S.D.W.). \\ Jiang Liu: 0000-0001-9933-0218 \\ Wenzhu Liu: 0000-0002-3935-1380 \\ Erkan Aydin: 0000-0002-8849-2788 \\ Stefaan De Wolf: 0000-0003-1619-9061
}

\section{Conflict of Interest}

The authors declare no conflict of interest.

\section{Acknowledgements}

This work was supported by the King Abdullah University of Science and Technology (KAUST) Office of Sponsored Research (OSR) under Award No: OSR-CARF URF/1/3079- 
33-01.

\section{Reference}

1. NREL, Best Research-Cell Efficiency Chart, https://www.nrel.gov/pv/cell-efficiency.html.

2. De Wolf, S.; Holovsky, J.; Moon, S. J.; Loper, P.; Niesen, B.; Ledinsky, M.; Haug, F. J.; Yum, J. H.; Ballif, C. Organometallic Halide Perovskites: Sharp Optical Absorption Edge and Its Relation to Photovoltaic Performance. J. Phys. Chem. Lett. 2014, 5, 1035-1039.

3. Ledinsky, M.; Schonfeldova, T.; Holovsky, J.; Aydin, E.; Hajkova, Z.; Landova, L.; Neykova, N.; Fejfar, A.; De Wolf, S. Temperature Dependence of the Urbach Energy in Lead Iodide Perovskites. J. Phys. Chem. Lett. 2019, 10, 1368-1373.

4. Yun, J. S.; Ho-Baillie, A.; Huang, S.; Woo, S. H.; Heo, Y.; Seidel, J.; Huang, F.; Cheng, Y. B.; Green, M. A. Benefit of Grain Boundaries in Organic-Inorganic Halide Planar Perovskite Solar Cells. J. Phys. Chem. Lett. 2015, 6, 875-880.

5. Yin, W.-J.; Shi, T.; Yan, Y. Unusual defect physics in $\mathrm{CH}_{3} \mathrm{NH}_{3} \mathrm{Pbl}_{3}$ perovskite solar cell absorber. Appl. Phys. Lett. 2014, 104, 063903.

6. Zhang, L.; Liu, C.; Wang, X.; Tian, Y.; Jen, A. K. Y.; Xu, B. Side-Chain Engineering on Dopant - Free Hole - Transporting Polymers toward Highly Efficient Perovskite Solar Cells (20.19\%). Adv. Funct. Mater. 2019, 29, 1904856.

7. Yang, S. J.; Kim, M.; Ko, H.; Sin, D. H.; Sung, J. H.; Mun, J.; Rho, J.; Jo, M. H.; Cho, K. Visualization and Investigation of Charge Transport in Mixed-Halide Perovskite via Lateral-Structured Photovoltaic Devices. Adv. Funct. Mater. 2018, 28, 1804067.

8. Zalar, P.; Kuik, M.; Henson, Z. B.; Woellner, C.; Zhang, Y.; Sharenko, A.; Bazan, G. C.; Nguyen, T. Q. Increased mobility induced by addition of a Lewis acid to a Lewis basic conjugated polymer. Adv. Mater. 2014, 26, 724-727.

9. Luo, D.; Yang, W.; Wang, Z.; Sadhanala, A.; Hu, Q.; Su, R.; Shivanna, R.; Trindade, G. F.; Watts, J. F.; Xu, Z.; Liu, T.; Chen, K.; Ye, F.; Wu, P.; Zhao, L.; Wu, J.; Tu, Y.; Zhang, Y.; Yang, X.; Zhang, W.; Friend, R. H.; Gong, Q.; Snaith, H. J.; Zhu, R. Enhanced photovoltage for inverted planar heterojunction perovskite solar cells. Science 2018, 360, 1442-1446.

10. Caliò, L.; Salado, M.; Kazim, S.; Ahmad, S. A Generic Route of Hydrophobic Doping in Hole Transporting Material to Increase Longevity of Perovskite Solar Cells. Joule 2018, 2, 1800-1815.

11. Gu, X.; Li, Y.; Mu, Y.; Zhang, M.; Lu, T.; Wang, P. FeCl 3 as a low-cost and efficient p-type dopant of SpiroOMeTAD for high performance perovskite solar cells. RSC Adv. 2018, 8, 9409-9413.

12. Wang, X.; Wu, J.; Yang, Y.; Liu, X.; Guo, Q.; Song, Z.; Li, G.; Lan, Z.; Huang, M. High performance and stable perovskite solar cells using vanadic oxide as a dopant for spiro-OMeTAD. J. Mater. Chem. A 2019, 7, 13256-13264.

13. Koh, T. M.; Dharani, S.; Li, H.; Prabhakar, R. R.; Mathews, N.; Grimsdale, A. C.; Mhaisalkar, S. G. Cobalt dopant with deep redox potential for organometal halide hybrid solar cells. ChemSusChem 2014, 7, 1909-1914.

14. Wang, S.; Yuan, W.; Meng, Y. S. Spectrum-Dependent Spiro-OMeTAD Oxidization Mechanism in Perovskite Solar Cells. ACS Appl. Mater. Interfaces 2015, 7, 24791-24798.

15. Hawash, Z.; Ono, L. K.; Qi, Y. Moisture and Oxygen Enhance Conductivity of LiTFSI-Doped Spiro-MeOTAD 
Hole Transport Layer in Perovskite Solar Cells. Adv. Mater. Interfaces 2016, 3, 1600117.

16. Abate, A.; Leijtens, T.; Pathak, S.; Teuscher, J.; Avolio, R.; Errico, M. E.; Kirkpatrik, J.; Ball, J. M.; Docampo, P.; McPherson, I.; Snaith, H. J. Lithium salts as "redox active" p-type dopants for organic semiconductors and their impact in solid-state dye-sensitized solar cells. Phys. Chem. Chem. Phys. 2013, 15, 2572-2579.

17. Xi, H.; Tang, S.; Ma, X.; Chang, J.; Chen, D.; Lin, Z.; Zhong, P.; Wang, H.; Zhang, C. Performance Enhancement of Planar Heterojunction Perovskite Solar Cells through Tuning the Doping Properties of Hole-Transporting Materials. ACS Omega 2017, 2, 326-336.

18. Li, J.; Zhang, G.; Holm, D. M.; Jacobs, I. E.; Yin, B.; Stroeve, P.; Mascal, M.; Moulé, A. J. Introducing Solubility Control for Improved Organic P-Type Dopants. Chem. Mater. 2015, 27, 5765-5774.

19. Wang, S.; Huang, Z.; Wang, X.; Li, Y.; Gunther, M.; Valenzuela, S.; Parikh, P.; Cabreros, A.; Xiong, W.; Meng, Y. S. Unveiling the Role of tBP-LiTFSI Complexes in Perovskite Solar Cells. J. Am. Chem. Soc. 2018, 140, 16720-16730.

20. Hawash, Z.; Ono, L. K.; Raga, S. R.; Lee, M. V.; Qi, Y. Air-Exposure Induced Dopant Redistribution and Energy Level Shifts in Spin-Coated Spiro-MeOTAD Films. Chem. Mater. 2015, 27, 562-569.

21. Wang, S.; Sina, M.; Parikh, P.; Uekert, T.; Shahbazian, B.; Devaraj, A.; Meng, Y. S. Role of 4-tertButylpyridine as a Hole Transport Layer Morphological Controller in Perovskite Solar Cells. Nano Lett. 2016, 16, 5594-5600.

22. Wang, Y.; Sun, K.; Xu, H.; Zhang, H.; Zhang, J.; Zhu, Y.; Jia, R.; Hu, Z. Origination of Anomalous Current Fluctuation in Perovskite Solar Cells. ACS Appl. Energy Mater. 2019, 2, 8138-8144.

23. Xu, B.; Zhu, Z.; Zhang, J.; Liu, H.; Chueh, C.-C.; Li, X.; Jen, A. K. Y. 4-Tert-butylpyridine Free Organic Hole Transporting Materials for Stable and Efficient Planar Perovskite Solar Cells. Adv. Energy Mater. 2017, 7, 1700683.

24. Liu, J.; Wu, Y.; Qin, C.; Yang, X.; Yasuda, T.; Islam, A.; Zhang, K.; Peng, W.; Chen, W.; Han, L. A dopantfree hole-transporting material for efficient and stable perovskite solar cells. Energy Environ. Sci. 2014, 7, 2963.

25. Yin, X.; Zhou, J.; Song, Z.; Dong, Z.; Bao, Q.; Shrestha, N.; Bista, S. S.; Ellingson, R. J.; Yan, Y.; Tang, W. Dithieno[3,2-b:2',3'-d]pyrrol-Cored Hole Transport Material Enabling Over 21\% Efficiency Dopant-Free Perovskite Solar Cells. Adv. Funct. Mater. 2019, 29, 1904300.

26. Luo, J. S.; Xia, J. X.; Yang, H.; Chen, L. L.; Wan, Z. Q.; Han, F.; Malik, H. A.; Zhu, X. H.; Jia, C. Y. Toward high-efficiency, hysteresis-less, stable perovskite solar cells: unusual doping of a hole-transporting material using a fluorine-containing hydrophobic Lewis acid. Energy Environ. Sci. 2018, 11, 2035-2045.

27. Welch, G. C.; Coffin, R.; Peet, J.; Bazan, G. C. Band gap control in conjugated oligomers via Lewis acids. J. Am. Chem. Soc. 2009, 131, 10802-10803.

28. Ye, T.; Chen, W.; Jin, S.; Hao, S.; Zhang, X.; Liu, H.; He, D. Enhanced Efficiency of Planar Heterojunction Perovskite Solar Cells by a Light Soaking Treatment on Tris(pentafluorophenyl)borane-Doped Poly(triarylamine) Solution. ACS Appl. Mater. Interfaces 2019, 11, 14004-14010.

29. Ye, T.; Wang, J.; Chen, W.; Yang, Y.; He, D. Improved Performance and Reproducibility of Perovskite Solar Cells by Well-Soluble Tris(pentafluorophenyl)borane as a p-Type Dopant. ACS Appl. Mater. Interfaces 2017, 9, 17923-17931.

30. Li, Z.; Xiao, C.; Yang, Y.; Harvey, S. P.; Kim, D. H.; Christians, J. A.; Yang, M.; Schulz, P.; Nanayakkara, S. U.; Jiang, C.-S.; Luther, J. M.; Berry, J. J.; Beard, M. C.; Al-Jassim, M. M.; Zhu, K. Extrinsic ion migration in perovskite solar cells. Energy Environ. Sci. 2017, 10, 1234-1242.

31. Liu, Y.; Hu, Y.; Zhang, X.; Zeng, P.; Li, F.; Wang, B.; Yang, Q.; Liu, M. Inhibited aggregation of lithium salt in spiro-OMeTAD toward highly efficient perovskite solar cells. Nano Energy 2020, 70, 104483. 
32. Li, Z.; Tinkham, J.; Schulz, P.; Yang, M.; Kim, D. H.; Berry, J.; Sellinger, A.; Zhu, K. Acid Additives Enhancing the Conductivity of Spiro-OMeTAD Toward High-Efficiency and Hysteresis-Less Planar Perovskite Solar Cells. Adv. Energy Mater. 2017, 7, 1601451.

33. Dang, H. X.; Wang, K.; Ghasemi, M.; Tang, M.-C.; De Bastiani, M.; Aydin, E.; Dauzon, E.; Barrit, D.; Peng, J.; Smilgies, D.-M.; De Wolf, S.; Amassian, A. Multi-cation Synergy Suppresses Phase Segregation in Mixed-Halide Perovskites. Joule 2019, 3, 1746-1764.

34. Wang, K.; Tang, M. C.; Dang, H. X.; Munir, R.; Barrit, D.; De Bastiani, M.; Aydin, E.; Smilgies, D. M.; De Wolf, S.; Amassian, A. Kinetic Stabilization of the Sol-Gel State in Perovskites Enables Facile Processing of High-Efficiency Solar Cells. Adv. Mater. 2019, 31, 1808357.

35. Saliba, M.; Correa-Baena, J.-P.; Wolff, C. M.; Stolterfoht, M.; Phung, N.; Albrecht, S.; Neher, D.; Abate, A. How to Make over 20\% Efficient Perovskite Solar Cells in Regular ( $-i-p)$ and Inverted $(p-i-n)$ Architectures. Chem. Mater. 2018, 30, 4193-4201.

36. Peng, J.; Khan, J. I.; Liu, W. Z.; Ugur, E.; Duong, T.; Wu, Y. L.; Shen, H. P.; Wang, K.; Dang, H.; Aydin, E.; Yang, X. B.; Wan, Y. M.; Weber, K. J.; Catchpole, K. R.; Laquai, F.; De Wolf, S.; White, T. P. A Universal Double-Side Passivation for High Open-Circuit Voltage in Perovskite Solar Cells: Role of Carbonyl Groups in Poly(methyl methacrylate). Adv. Energy Mater. 2018, 8, 1801208.

37. Jiang, Q.; Zhang, L.; Wang, H.; Yang, X.; Meng, J.; Liu, H.; Yin, Z.; Wu, J.; Zhang, X.; You, J. Enhanced electron extraction using $\mathrm{SnO}_{2}$ for high-efficiency planar-structure $\mathrm{HC}\left(\mathrm{NH}_{2}\right)_{2} \mathrm{Pbl}_{3}$-based perovskite solar cells. Nat. Energy 2016, 1, 16177.

38. Fernandez Garrillo, P. A.; Grevin, B.; Chevalier, N.; Borowik, L. Calibrated work function mapping by Kelvin probe force microscopy. Rev. Sci. Instrum. 2018, 89, 043702.

39. Schulz, P.; Edri, E.; Kirmayer, S.; Hodes, G.; Cahen, D.; Kahn, A. Interface energetics in organo-metal halide perovskite-based photovoltaic cells. Energy Environ. Sci. 2014, 7, 1377-1381.

40. Nguyen, W. H.; Bailie, C. D.; Unger, E. L.; McGehee, M. D. Enhancing the hole-conductivity of spiroOMeTAD without oxygen or lithium salts by using spiro(TFSI)(2) in perovskite and dye-sensitized solar cells. J. Am. Chem. Soc. 2014, 136, 10996-11001.

41. Tan, B.; Raga, S. R.; Chesman, A. S. R.; Fürer, S. O.; Zheng, F.; McMeekin, D. P.; Jiang, L.; Mao, W.; Lin, X.; Wen, X.; Lu, J.; Cheng, Y. B.; Bach, U. LiTFSI-Free Spiro-OMeTAD-Based Perovskite Solar Cells with Power Conversion Efficiencies Exceeding 19\%. Adv. Energy Mater. 2019, 9, 1901519.

42. Abate, A.; Saliba, M.; Hollman, D. J.; Stranks, S. D.; Wojciechowski, K.; Avolio, R.; Grancini, G.; Petrozza, A.; Snaith, H. J. Supramolecular halogen bond passivation of organic-inorganic halide perovskite solar cells. Nano Lett. 2014, 14, 3247-3254.

43. Qian, F.; Yuan, S.; Cai, Y.; Han, Y.; Zhao, H.; Sun, J.; Liu, Z.; Liu, S. Novel Surface Passivation for Stable $\mathrm{FA}_{0.85} \mathrm{MA}_{0.15} \mathrm{Pbl}_{3}$ Perovskite Solar Cells with 21.6\% Efficiency. Solar RRL 2019, 3, 1900072.

44. Aydin, E.; De Bastiani, M.; De Wolf, S. Defect and Contact Passivation for Perovskite Solar Cells. Adv. Mater. 2019, 31, 1900428.

45. Yang, Z.; Dou, J.; Kou, S.; Dang, J.; Ji, Y.; Yang, G.; Wu, W. Q.; Kuang, D. B.; Wang, M. Multifunctional Phosphorus-Containing Lewis Acid and Base Passivation Enabling Efficient and Moisture-Stable Perovskite Solar Cells. Adv. Funct. Mater. 2020, 30, 1910710.

46. Domanski, K.; Correa-Baena, J. P.; Mine, N.; Nazeeruddin, M. K.; Abate, A.; Saliba, M.; Tress, W.; Hagfeldt, A.; Gratzel, M. Not All That Glitters Is Gold: Metal-Migration-Induced Degradation in Perovskite Solar Cells. ACS Nano 2016, 10, 6306-6314.

47. Arora, N.; Dar, M. I.; Hinderhofer, A.; Pellet, N.; Schreiber, F.; Zakeeruddin, S. M.; Gratzel, M. Perovskite solar cells with CuSCN hole extraction layers yield stabilized efficiencies greater than $20 \%$. Science $\mathbf{2 0 1 7}$, 
$358,768-771$.

11 


\section{Table of Contents (TOC)}

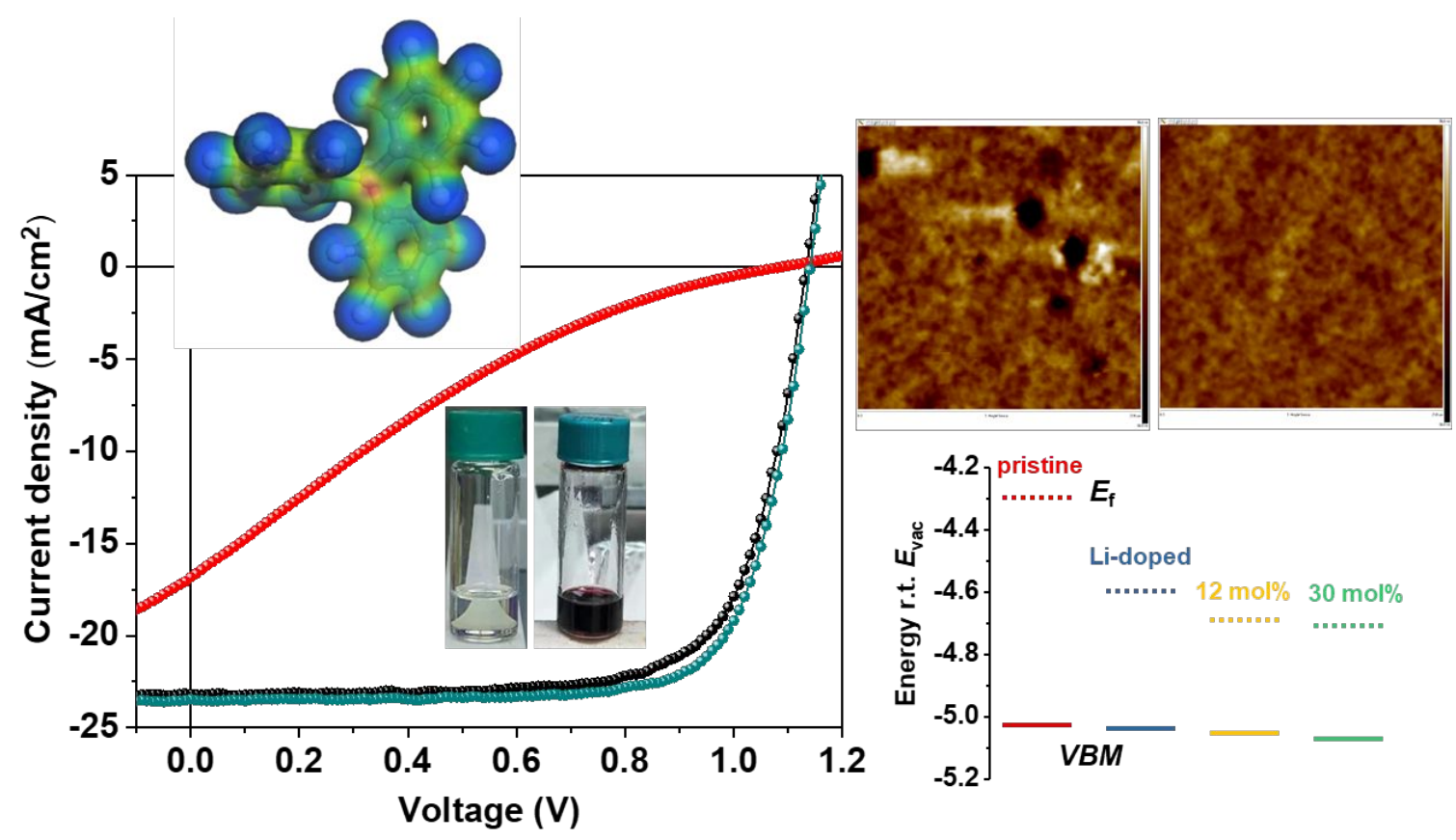

